\title{
$(s, S)$-TYPE POLICY \\ FOR A PRODUCTION INVENTORY PROBLEM WITH LIMITED BACKLOGGING AND WITH STOCKOUTS
}

\begin{abstract}
A production inventory problem with limited backlogging and with stockouts is described in a discrete time, stochastic optimal control framework with finite horizon. It is proved by dynamic programming methods that an optimal policy is of $(s, S)$-type. This means that in every period the policy is completely determined by two fixed levels of the stochastic inventory process considered.
\end{abstract}

1. Introduction. We discuss a dynamic, stochastic inventory model with limited backlogging and with stockouts when the inventory process crosses the backlogging limits. Usually in the literature both these situations are discussed in separate models ([6], [2], [1]). Our paper joins the models, as is done in Bylka [3] for the deterministic case. The negative levels of the inventory process are limited. In every period the limit results in that only a part of the excess of demand over supply is backlogged and satisfied when additional inventory becomes available. The remaining part cannot be satisfied and is completely lost. Both cases are considered in the shortage cost structure. The costs of shortage, storage and ordering (ordering being the sum of production and setup costs) are included in the objective functional.

2. Formulation of the problem. We describe the problem in the optimal control framework.

Let $N$ be a positive integer and let $\left\{W_{t}: t=0,1, \ldots, N-1\right\}$ denote a sequence of independent, identically distributed, nonnegative random variables with finite expected value $\left(E\left(W_{t}\right)<\infty\right)$. The variables represent the

1991 Mathematics Subject Classification: Primary $90 \mathrm{~B} 05$.

Key words and phrases: inventory, limited backlogging, dynamic programming, $(s, S)$ policy, $k$-convexity.

This research is partially supported by KBN grant. No. 1 H02B00410. 
random demands which appear at the ends of periods $t=0,1, \ldots, N-1$. The inventory process $\left\{X_{t}: t=0,1, \ldots, N\right\}$ is given by the following realvalued decision process:

$$
\begin{aligned}
X_{0} & =x_{0}, \\
X_{t+1} & =\max \left(-\beta_{t}, X_{t}+u_{t}\left(X_{t}\right)-W_{t}\right), \quad t=0,1, \ldots, N-1,
\end{aligned}
$$

where $\beta_{t} \geq 0, u_{t}: \mathbb{R} \rightarrow \mathbb{R}^{+}$is a Borel-measurable decision (control) function and $x_{0} \in \mathbb{R}$.

In this description $x_{0}$ is the initial level of the inventory process, $\beta_{t}$ limits the backlogging and $u_{t}$ denotes the order (or production) decision. We assume that the order decisions are made and the ordered goods delivered immediately at the beginning of each period. The demands are supposed to occur at the end of each period after the orders have entered the stock. The limit $\beta_{t}$ makes the shortages of size up to $\beta_{t}$ allowed and backordered. The shortages of size over $\beta_{t}$ are completely lost (stockouts).

A sequence of decision functions

$$
\pi=\left(u_{0}(\cdot), u_{1}(\cdot), \ldots, u_{N-1}(\cdot)\right)
$$

is called a policy (strategy). The model considers some costs connected with any strategy and corresponding process $(2.1)$. The costs satisfy the following assumptions:

(2.2) Assumptions. (i) The ordering cost function at the period $t, t=$ $0,1, \ldots, N-1$, is given by

$$
c_{t}(u)= \begin{cases}K_{t}+c_{t} u & \text { if } u>0, \\ 0 & \text { if } u=0,\end{cases}
$$

where $K_{t} \geq 0, c_{t} \geq 0 . K_{t}$ denotes the setup cost and $c_{t}$ denotes the unit production cost.

(ii) $\left\{K_{t}\right\}$ is such that

$$
K_{t} \geq K_{t+1} \quad \text { for } t=0,1, \ldots, N-2 .
$$

(iii) For every period $t, t=0,1, \ldots, N-1$, the inventory cost function $h_{t}: \mathbb{R}^{+} \rightarrow \mathbb{R}^{+}$is convex and nondecreasing with $h_{t}(x) \leq \bar{h}_{t}(1+x)$ for all $x \in \mathbb{R}^{+}$and some $\bar{h}_{t} \in \mathbb{R}^{+}$.

(iv) For every period $t, t=0,1, \ldots, N-1$, the shortage cost function $p_{t}: \mathbb{R}^{+} \rightarrow \mathbb{R}^{+}$is convex and nondecreasing with $p_{t}(0)=0, p_{t}(x) \leq \bar{p}_{t}(1+x)$ for all $x \in \mathbb{R}^{+}$and some $\bar{p}_{t} \in \mathbb{R}^{+}$. Additionally, $E\left(p_{t}^{\prime+}\left(W_{t}\right)\right)>c_{t}$ and $p_{t}^{\prime+}\left(\beta_{t}\right)-p_{t}^{\prime-}\left(\beta_{t}\right)>c_{t+1}$, where $E$ denotes the expectation with respect to the probability distribution of $W_{t}$ and $p^{+}$and $p_{t}^{\prime-}$ denote the right- and left-hand derivatives. If $\beta_{t}=0$ then we put $p_{t}^{\prime-}(0)=0$.

The objective functional to be minimized is the expected value of all the cost incurred over the horizon 


$$
\begin{array}{r}
J(\pi)=\underset{\left\{W_{t}\right\}}{\mathbb{E}}\left(\sum _ { t = 0 } ^ { N - 1 } \left\{c_{t}\left(u_{t}\left(X_{t}\right)\right)+h_{t}\left(\max \left(0, X_{t}+u_{t}\left(X_{t}\right)-W_{t}\right)\right)\right.\right. \\
\left.\left.+p_{t}\left(\max \left(0, W_{t}-X_{t}-u_{t}\left(X_{t}\right)\right)\right)\right\}\right) .
\end{array}
$$

We are looking for a strategy $\pi^{*}$ (optimal) such that

$$
J\left(\pi^{*}\right)=\min _{\pi} J(\pi) .
$$

Remarks on the model. Observe that for $\beta_{t}=\infty, t=0,1, \ldots, N-1$, all the negative levels of the inventory process (2.1) are admissible. This means that an unsatisfied demand is completely backlogged. If $\beta_{t}=0$, $t=0,1, \ldots, N-1$, then only positive levels of the inventory process (2.1) are allowed. There is no backlogging and so unsatisfied demands are completely lost (stockouts). It is known ([6], [2], [1], [4], [5]) that in both these cases the optimal strategies are of $(s, S)$-type. This means that in every period $t$ the decision function $u_{t}^{*}$ is determined by two inventory levels $s_{t}$ and $S_{t}$ such that

$$
u_{t}^{*}(x)= \begin{cases}S_{t}-x & \text { if } x \leq s_{t}, \\ 0 & \text { if } x>s_{t} .\end{cases}
$$

The aim of this paper is to generalize the result for the case $\beta_{t} \geq 0$, $\beta_{t}<\infty, t=0,1, \ldots, N-1$.

Remarks on the Assumptions (2.2). In the present model the shortages of size up to $\beta_{t}$ and over $\beta_{t}, t=0,1, \ldots, N-1$, are differently treated. This fact is included into the structure of the shortage cost $p_{t}$. The discontinuity of $p_{t}^{\prime}$ at $\beta_{t}$ assumed in (2.2)(iv) means that the rate of growth of the shortage cost above the limit $\beta_{t}$ is substantially greater than below $\beta_{t}$. The size of the jump is greater than the next period marginal production cost $c_{t+1}$. The remaining assumptions in (2.2) are of the type similar to those which appeared in the discussion of the case $\beta_{t}=0, t=0,1, \ldots, N-1([1, \S \mathrm{IV}$, Sec. IV]).

3. Auxiliary theorem and lemmas. Here and in the next sections we apply dynamic programming methods to determine an optimal strategy $\pi^{*}$.

Theorem 3.1 [7, Theorem 1.1]. Let $V_{0}, V_{1}, \ldots, V_{N}$ be nonnegative measurable functions defined on $\mathbb{R}$ such that

$$
\begin{aligned}
V_{N} \equiv & 0, \\
V_{t}(x)= & \inf _{u \geq 0}\left\{c_{t}(u)+E\left(h_{t}\left(\max \left(0, u+x-W_{t}\right)\right)\right)\right. \\
& +E\left(p_{t}\left(\max \left(0, W_{t}-x-u\right)\right)\right) \\
& \left.+E\left(V_{t+1}\left(\max \left(-\beta_{t}, x+u-W_{t}\right)\right)\right)\right\}, \quad t=N-1, \ldots, 0 .
\end{aligned}
$$


If $\pi^{*}=\left(u_{0}^{*}, u_{1}^{*}, \ldots, u_{N-1}^{*}\right)$ is a strategy such that

$$
\begin{aligned}
V_{t}(x)= & c_{t}\left(u_{t}^{*}(x)\right)+E\left(h_{t}\left(\max \left(0, u_{t}^{*}(x)+x-W_{t}\right)\right)\right) \\
& +E\left(p_{t}\left(\max \left(0, W_{t}-u_{t}^{*}(x)-x\right)\right)\right) \\
& +E\left(V_{t+1}\left(\max \left(-\beta_{t}, u_{t}^{*}(x)+x-W_{t}\right)\right)\right)
\end{aligned}
$$

then $\pi^{*}$ is optimal for the problem (2.1)-(2.4).

It is convenient to rewrite the assumptions of Theorem 3.1 in a slightly different form. For this put $y=x+u$ and

$$
\begin{aligned}
G_{t}(y)= & c_{t} y+E\left(h_{t}\left(\max \left(0, y-W_{t}\right)\right)\right)+E\left(p_{t}\left(\max \left(0, W_{t}-y\right)\right)\right) \\
& +E\left(V_{t+1}\left(\max \left(-\beta_{t}, y-W_{t}\right)\right)\right) .
\end{aligned}
$$

Using this notation and the definition of $c_{t}(\cdot)$ the equations (3.1) may be written as

$$
V_{N}(x)=0, \quad V_{t}(x)=\inf _{y \geq x}\left\{K_{t} \chi_{\{y>x\}}+G_{t}(y)-c_{t} x\right\}
$$

where

$$
t=N-1, \ldots, 0, \quad \chi_{\{y>x\}}= \begin{cases}1 & \text { if } y>x \\ 0 & \text { if } y=x\end{cases}
$$

Moreover, if $y_{t}^{*}(x)$ is such that

$$
V_{t}(x)= \begin{cases}K_{t}+G_{t}\left(y_{t}^{*}(x)\right)-c_{t} x & \text { if } y_{t}^{*}(x)>x, \\ G_{t}\left(y_{t}^{*}(x)\right)-c_{t} x & \text { if } y_{t}^{*}(x)=x,\end{cases}
$$

then

$$
u_{t}^{*}(x)=y_{t}^{*}(x)-x .
$$

Observe that the right-hand sides of (3.2) for $t=N-1, \ldots, 0$ are of the type

$$
\inf _{y \geq x}\left\{K \chi_{\{y>x\}}+G(y)-c x\right\}, \quad x \in \mathbb{R}
$$

where $K \geq 0, G: \mathbb{R} \rightarrow \mathbb{R}$ and $c>0$. Therefore we start the discussion with the auxiliary problem (3.4). The problem means that for every $x \in \mathbb{R}$ we are looking for $y^{*}(x)$ such that

$$
K \chi_{\left\{y^{*}(x)>x\right\}}+G\left(y^{*}(x)\right)-c x=\inf _{y \geq x}\left\{K \chi_{\{y>x\}}+G(y)-c x\right\} .
$$

For this the notion of $k$-convexity will be useful.

Definition 3.1 [6]. A function $f: \mathbb{R} \rightarrow \mathbb{R}$ is said to be $k$-convex, $k \in \mathbb{R}^{+}$, if it satisfies

$$
k+f(y+z) \geq f(y)+z \frac{f(y)-f(y-b)}{b} \quad \text { for all } z \geq 0, b>0 \text { and } y \in \mathbb{R} .
$$

Remark 3.1. It follows from the definition that a convex function is 0 -convex; a $k$-convex function is also $l$-convex for any $l \geq k$; the sum of a 
$k$-convex function and an $l$-convex function is $(k+l)$-convex; if $f$ is $k$-convex and $W$ is a random variable such that for every $x, E|f(x-W)|<\infty$ then $x \rightarrow E(f(x-W))$ is also $k$-convex.

Lemma 3.1 [1, pp. 318-320]. Consider (3.4). Let $G$ be K-convex, continuous and such that $G(y) \rightarrow \infty$ as $|y| \rightarrow \infty$. Then there exist numbers $s$, $S, s \leq S$, such that

(i) $G(S)=\inf _{y \in \mathbb{R}} G(y)$,

(ii) $G(s)=K+G(S)$,

(iii) $G(x) \leq K+G(y)$ for all $x, y$ with $s \leq x \leq y$,

(iv) the function

is a solution of (3.4),

$$
y^{*}(x)= \begin{cases}S & \text { if } x \leq s, \\ x & \text { if } x>s\end{cases}
$$

(v) the function

$$
V(x)=\inf _{y \geq x}\left\{K \chi_{\{y>x\}}+G(y)-c x\right\}= \begin{cases}K+G(S)-c x & \text { if } x \leq s, \\ G(x)-c x & \text { if } x>s\end{cases}
$$

is continuous and $K$-convex.

Proof. The proof of (i)-(iii) is given by Bensoussan et al. [1, pp. 318319]. The proof of (iv)-(v) follows from the proof of Proposition 8.2 in [1, pp. 319-320] and from the fact that the sum of a $K$-convex function and a linear function is $K$-convex.

Remark 3.2. Observe that if $G(x) \leq \bar{G}(1+|x|)$ for some $\bar{G}$ then $V(x) \leq \bar{V}(1+|x|)$ for some $\bar{V}$.

An optimal strategy for (2.1)-(2.4) is determined by Theorem 3.1 and remarks (3.3) and (3.2). So the second part of this section is connected with $p_{t}, h_{t}$ describing $G_{t}$ in $(3.2)$.

Put

and

$$
\left(l p_{t}\right)(z)=p_{t}^{\prime-}\left(\beta_{t}\right)\left(z-\beta_{t}\right)+p_{t}\left(\beta_{t}\right)
$$

Moreover, let

$$
P_{t}(z)= \begin{cases}p_{t}(z) & \text { for } z \in\left[0, \beta_{t}\right) \\ \left(l p_{t}\right)(z) & \text { for } z \in\left[\beta_{t}, \infty\right)\end{cases}
$$

$$
g_{t}(z)= \begin{cases}0 & \text { for } z \in\left[0, \beta_{t}\right) \\ p_{t}(z)-\left(l p_{t}\right)(z) & \text { for } z \in\left[\beta_{t}, \infty\right)\end{cases}
$$

Remark 3.3. Observe that the assumptions (2.2)(iv) imply that

(i) $P_{t}$ and $g_{t}$ are convex, nonnegative and nondecreasing on $\mathbb{R}^{+}$,

(ii) $P_{t}(z) \leq p_{t}(z) \leq \overline{\bar{p}}_{t}(1+z)$, for $z \in \mathbb{R}^{+}$and some $\overline{\bar{p}}_{t} \in \mathbb{R}, \overline{\bar{p}}_{t} \leq \bar{p}_{t}$,

(iii) $g_{t}(z) \leq \bar{g}_{t}(1+z)$ with $\bar{g}_{t} \leq \bar{p}_{t}$ and $z \in \mathbb{R}^{+}$. 
The assumption $p_{t}^{\prime+}\left(\beta_{t}\right)-p_{t}^{\prime-}\left(\beta_{t}\right)>c_{t+1}$ gives

(iv) $g^{\prime+}\left(\beta_{t}\right)>c_{t+1}$.

Define

$$
l_{t}(y)=c_{t} y+E\left(h_{t}\left(\max \left(0, y-W_{t}\right)\right)\right)+E\left(P_{t}\left(\max \left(0, W_{t}-y\right)\right)\right)
$$

and let

$$
\begin{aligned}
L_{t}(y) & =l_{t}(y)+E\left(g_{t}\left(\max \left(0, W_{t}-y\right)\right)\right) \\
& =c_{t} y+E\left(p_{t}\left(\max \left(0, W_{t}-y\right)\right)\right)+E\left(h_{t}\left(\max \left(0, y-W_{t}\right)\right)\right) .
\end{aligned}
$$

Now we establish useful properties of $l_{t}$ and $L_{t}$.

Lemma 3.2. (i) $l_{t}$ and $L_{t}$ are convex functions,

(ii) there exists $\bar{L}_{t}$ such that $L_{t}(y) \leq \bar{L}_{t}(1+|y|)$,

(iii) $L_{t}(y) \rightarrow \infty$ as $|y| \rightarrow \infty$.

Proof. First observe that the functions defined by

$$
\begin{aligned}
\widetilde{P}_{t}(y) & =P_{t}(\max (0,-y)), \\
\widetilde{h}_{t}(y) & =h_{t}(\max (0, y)), \quad \widetilde{g}_{t}(y)=g_{t}(\max (0,-y))
\end{aligned}
$$

are convex, as superpositions of convex and convex nondecreasing functions. Secondly, for all $y, E\left|\widetilde{P}_{t}\left(y-W_{t}\right)\right|<\infty, E\left|\widetilde{h}_{t}\left(y-W_{t}\right)\right|<\infty$ and $E\left|\widetilde{g}_{t}\left(y-W_{t}\right)\right|<\infty$ because $E\left(W_{t}\right)<\infty$ and $h_{t}, g_{t}, P_{t}$ are bounded by linear functions (assumptions (2.2)(ii), Remark 3.3(ii)-(iii)). So by the last part of Remark 3.1 the functions $E\left(\widetilde{P}_{t}\left(y-W_{t}\right)\right), E\left(\widetilde{h}_{t}\left(y-W_{t}\right)\right)$ and $E\left(\widetilde{g}_{t}\left(y-W_{t}\right)\right)$ are convex with respect to $y$, which, together with the definitions (3.7) and (3.8), gives (i).

The assertion (ii) follows from the assumption that $p_{t}, h_{t}$ are bounded by linear functions.

For the proof of (iii) note that

(a) $E\left(p_{t}\left(\max \left(0, W_{t}-y\right)\right) \geq p_{t}(0)\right.$ and $E\left(h_{t}\left(\max \left(0, y-W_{t}\right)\right)\right) \geq h_{t}(0)$. Hence by $(3.8), L_{t}(y) \geq c_{t} y+p_{t}(0)+h_{t}(0) \rightarrow \infty$ as $y \rightarrow \infty$.

(b) Consider $y<0, y \rightarrow-\infty$. So $p_{t}\left(\max \left(0, W_{t}-y\right)\right)=p_{t}\left(W_{t}-y\right) \geq$ $p_{t}^{+}\left(W_{t}\right)(-y)+p_{t}\left(W_{t}\right)$ because $p_{t}$ is a convex function. Hence by (3.8),

$$
L_{t}(y) \geq h_{t}(0)+p_{t}(0)+\left(E\left(p_{t}^{\prime+}\left(W_{t}\right)\right)-c_{t}\right)(-y) \rightarrow \infty
$$

as $y \rightarrow-\infty$ because by assumption (2.2)(iv), $E\left(p_{t}^{\prime+}\left(W_{t}\right)\right)-c_{t}>0$, which completes the proof.

In the next section we turn to the dynamic equations (3.2).

4. Optimal strategy. By induction we prove the following main result of the paper. 
THEOREM 4.1. Under the assumptions (2.2) there exists a sequence of pairs $\left(s_{t}, S_{t}\right)$ with $s_{t} \leq S_{t}, t=0,1, \ldots, N-1$, such that

$$
u_{t}^{*}(x)= \begin{cases}S_{t}-x & \text { for } x \leq s_{t} \\ 0 & \text { for } x>s_{t}\end{cases}
$$

is an optimal strategy for the problem (2.1)-(2.4).

Proof. Step 1. Consider $t=N-1$ and the dynamic equation of the form (3.2). Note that in this case $G_{N-1}=L_{N-1}$, where $L_{N-1}$ is given by (3.8). Thus, by Lemma 3.2,

(a) $G_{N-1}$ is a convex function and hence it is $K_{N-1}$-convex and continuous on $\mathbb{R}$,

(b) there exists $\bar{G}_{N-1}$ such that $G_{N-1}(y) \leq \bar{G}_{N-1}(1+|y|)$,

(c) $G_{N-1}(y) \rightarrow \infty$ as $|y| \rightarrow \infty$.

Hence by Lemma 3.1(iv) there exist $s_{N-1}$ and $S_{N-1}, s_{N-1} \leq S_{N-1}$, such that

$$
y_{N-1}^{*}(x)= \begin{cases}S_{N-1} & \text { if } x \leq s_{N-1}, \\ x & \text { if } x>s_{N-1}\end{cases}
$$

which by (3.3) gives the assertion of the theorem for $t=N-1$.

Step 2. Let $t=N-k, N-1 \geq k \geq 1$. We specify the induction assumptions:

(i) $G_{t}$ is $K_{t}$-convex and a continuous,

(ii) there exists $\bar{G}_{t}$ such that $G_{t}(y) \leq \bar{G}_{t}(1+|y|)$,

(iii) $G_{t}(y) \rightarrow \infty$ as $|y| \rightarrow \infty$.

Note that by (3.2) and Lemma 3.1 the induction assumptions imply that there exists a pair $\left(s_{t}, S_{t}\right)$ such that

$$
y_{t}^{*}(x)= \begin{cases}S_{t} & \text { if } x \leq s_{t} \\ x & \text { if } x>s_{t}\end{cases}
$$

and moreover, the assertion (v) of Lemma 3.1 shows that

$$
V_{t}(x)=-c_{t} x+ \begin{cases}K_{t}+G_{t}\left(S_{t}\right) & \text { if } x \leq s_{t}, \\ G_{t}(x) & \text { if } x>s_{t}\end{cases}
$$

is a continuous $K_{t}$-convex function. By the induction assumptions (i)-(iii) we have

(a) $G_{t}\left(S_{t}\right) \leq G_{t}(y), y \in \mathbb{R} ; G_{t}\left(s_{t}\right)=K_{t}+G_{t}\left(S_{t}\right)$;

(b) $G_{t}(y) \leq K_{t}+G_{t}(x)$ for all $x, y$ such that $s_{t} \leq y \leq x$.

Remark 3.2 says that there exists $\bar{V}_{t}$ such that

$$
V_{t}(x) \leq \bar{V}_{t}(1+|x|) \text {. }
$$


In order to prove the theorem it is sufficient to show that (i)-(iii) are satisfied for $G_{t-1}$. We recall the formulas for $G_{t-1}$ :

$$
\begin{aligned}
G_{t-1}(y)= & c_{t-1} y+E\left(h_{t-1}\left(\max \left(0, y-W_{t-1}\right)\right)\right) \\
& +E\left(p_{t-1}\left(\max \left(0, W_{t-1}-y\right)\right)\right) \\
& +E\left(V_{t}\left(\max \left(-\beta_{t-1}, y-W_{t-1}\right)\right)\right) \\
= & L_{t-1}(y)+E\left(V_{t}\left(\max \left(-\beta_{t-1}, y-W_{t-1}\right)\right)\right) \\
= & l_{t-1}(y)+E\left(g_{t-1}\left(\max \left(0, W_{t-1}-y\right)\right)\right) \\
& +E\left(V_{t}\left(\max \left(-\beta_{t-1}, y-W_{t-1}\right)\right)\right),
\end{aligned}
$$

where $l_{t-1}$ and $L_{t-1}$ are given by (3.7), (3.8).

Step 3. In this step we will show that

(a) $G_{t-1}(y) \rightarrow \infty$ as $|y| \rightarrow \infty$,

(b) $G_{t-1}$ is a continuous function on $\mathbb{R}$,

(c) there exists $\bar{G}_{t-1}$ such that $G_{t-1}(y) \leq \bar{G}_{t-1}(1+|y|)$.

For the proof of (a) we recall that by the definition (3.1), $V_{t} \geq 0$. So together by Lemma 3.2 (iii) we have $G_{t-1}(y) \geq L_{t-1}(y) \rightarrow \infty$ as $|y| \rightarrow \infty$.

For (b) observe that by Lemma $3.2(\mathrm{i}), L_{t-1}$ is convex on $\mathbb{R}$ and so continuous. Thus it is sufficient to show that the map $y \rightarrow E\left(V_{t}\left(\max \left(-\beta_{t-1}, y-\right.\right.\right.$ $\left.\left.W_{t-1}\right)\right)$ ) is continuous. Let $\left\{y_{n}\right\} \subset \mathbb{R}$ be a convergent sequence. Then (4.3) implies that

$$
V_{t}\left(\max \left(-\beta_{t-1}, y_{n}-W_{t-1}\right)\right) \leq \bar{V}_{t}\left(1+\sup \left|y_{n}\right|+W_{t-1}\right) .
$$

Observe that $E\left(\bar{V}_{t}\left(1+\sup \left|y_{n}\right|+W_{t-1}\right)\right)<\infty$ because $E\left(W_{t-1}\right)<\infty$. Thus the continuity follows from Lebesgue's theorem and the continuity of $V_{t}$.

The assertion (c) is a consequence of (4.3), Lemma 3.2(ii) and the assumption that $E\left(W_{t-1}\right)<\infty$.

For the proof of the theorem it remains to show that $G_{t-1}$ is $K_{t-1}$-convex.

This will be done in the next step and in the Appendix.

Step 4. Now it is convenient to write $G_{t-1}$ in the form

$$
G_{t-1}(y)=l_{t-1}(y)+R(y),
$$

where $R$ is defined as

$$
R(y)=E\left(g_{t-1}\left(\max \left(0, W_{t-1}-y\right)\right)\right)+E\left(V_{t}\left(\max \left(-\beta_{t-1}, y-W_{t-1}\right)\right)\right) .
$$

Lemma 3.2(i) states that $l_{t-1}$ is convex and so 0 -convex. Thus it is sufficient to prove that $R$ is $K_{t-1}$-convex.

Let

$$
r(y)=g_{t-1}(\max (0,-y))+V_{t}\left(\max \left(-\beta_{t-1}, y\right)\right) .
$$

Hence, $R(y)=E\left(r\left(y-W_{t-1}\right)\right)$ and by Remark 3.3(iii), (4.3) and the assumption that $E\left(W_{t-1}\right)<\infty$ we can assert that $E\left|r\left(y-W_{t-1}\right)\right|<\infty$. So by 
the last part of Remark 3.1 it is sufficient to show that $r(\cdot)$ is a $K_{t-1}$-convex function. This will be done in the Appendix and will finish the proof of this theorem.

Remarks to the proof. For the case $\beta_{t}=0, t=0,1, \ldots, N-1$, it was remarked by Bertsekas $[2$, p. 10$]$ that $K_{t}$-convexity of $G_{t}$ does not imply $K_{t^{-}}$ convexity of $E\left(V_{t}\left(\max \left(0, y-W_{t-1}\right)\right)\right)$. This explains the reason for which we introduce the function $g_{t-1}$ and prove the $K_{t-1}$-convexity of $R$. The argument is similar to that given by Bertsekas [2, pp. 105-106] and Bensoussan et al. [1, pp. 345-348].

5. Appendix. $K_{t-1}$-convexity of $r$. We recall that by (3.6) and Remark 3.3(iv),

$$
g_{t-1}(y)= \begin{cases}0 & \text { for } y \in\left[0, \beta_{t-1}\right) \\ p_{t-1}(y)-\left(l p_{t-1}\right)(y) & \text { for } y \in\left[\beta_{t-1}, \infty\right)\end{cases}
$$

is a convex function with

$$
g_{t-1}^{++}\left(\beta_{t-1}\right)>c_{t}
$$

Thus the definition of $g_{t-1}$ gives

$$
g_{t-1}(\max (0,-y))= \begin{cases}0 & \text { for } y \geq-\beta_{t-1} \\ g_{t-1}(-y) & \text { for } y<-\beta_{t-1}\end{cases}
$$

Notation. It is convenient in this section to drop some subscripts and put

$g_{t-1}=g, \quad \beta_{t-1}=\beta \quad$ and $\quad V_{t}=V, \quad G_{t}=G, \quad s_{t}=s, \quad S_{t}=S, \quad c_{t}=c$.

Let us start with the following

Lemma 5.1. For $x \geq-\beta$ we have

$$
K_{t}+V(x) \geq V(-\beta)-g^{\prime+}(\beta)(x+\beta) .
$$

P r o of. Consider three cases:

(i) $-\beta \leq s<x$. By (4.1), (4.2)(a) and (5.1) we obtain

$$
\begin{aligned}
K_{t}+V(x) & =K_{t}-c x+G(x) \geq K_{t}-c x+G(S) \\
& =V(-\beta)+c(-\beta)-c x=V(-\beta)-c(x+\beta) \\
& \geq V(-\beta)-g^{\prime+}(\beta)(\beta+x) .
\end{aligned}
$$

(ii) $-\beta \leq x \leq s$. Using (4.1) and (5.1) we get

$$
K_{t}+V(x)=2 K_{t}-c x+G(S) \geq K_{t}-c x+G(S)
$$

and the remaining part of the proof is as in (i). 
(iii) $s<-\beta \leq x$. By (4.1), (4.2)(b) and (5.1) we have

$$
\begin{aligned}
K_{t}+V(x) & =K_{t}-c x+G(x) \geq-c x+G(-\beta) \\
& =-c x+(V(-\beta)-c \beta) \geq V(-\beta)-g^{\prime+}(\beta)(x+\beta) .
\end{aligned}
$$

Now we are in a position to check the $K_{t-1}$-convexity (Definition 3.1 ) of $r$.

Proposition 5.1. The function $r$ given by (4.4) satisfies the inequality

$$
K_{t-1}+r(y+z) \geq r(y)+z \frac{r(y)-r(y-b)}{b}
$$

for all $y \in \mathbb{R}, z \geq 0, b>0$.

Proof. Let $y \in \mathbb{R}, z \geq 0$ and $b>0$. Consider four cases.

(i) $-\beta \leq y-b<y \leq y+z$. By (4.4) and (5.2),

$r(y+z)=0+V(y+z), \quad r(y)=0+V(y), \quad r(y-b)=0+V(y-b)$

and (5.3) is a consequence of the $K_{t}$-convexity of $V$ and the assumption (2.2)(ii) that $K_{t-1} \geq K_{t}$.

(ii) $y-b<y \leq y+z \leq-\beta$. Then (4.4) and (5.2) give $r(y+z)=$ $g(-y-z)+V(-\beta), r(y)=g(-y)+V(-\beta)$ and $r(y-b)=g(-(y-b))+V(-\beta)$. The inequality (5.3) follows from the convexity of $g(\max (0,-y))$.

(iii) $y-b<y \leq-\beta \leq y+z$. In this case $r(y+z)=0+V(y+z)$, $r(y)=g(-y)+V(-\beta)$ and $r(y-b)=g(-(y-b))+V(-\beta)$. So (5.3) and the assumptions $K_{t-1} \geq K_{t}$ and $g(\beta)=0$ make it sufficient to show that

$$
K_{t}+V(y+z) \geq g(-y)-g(\beta)+V(-\beta)+z \frac{g(-y)-g(-y+b)}{b},
$$

For this observe that from the convexity of $g$ it follows that

$$
g(\beta)-g(-y)=g(-y+(\beta+y))-g(-y) \geq g^{\prime+}(-y)(\beta+y)
$$

and

$$
g(-y+b)-g(-y) \geq g^{\prime+}(-y) b .
$$

Thus

$$
\begin{aligned}
g(-y)-g(\beta)+V(-\beta) & +z \frac{g(-y)-g(-y+b)}{b} \\
\leq & -g^{\prime+}(-y)(\beta+y)+V(-\beta)+\frac{z}{b}\left(-g^{\prime+}(-y) b\right) \\
= & V(-\beta)-g^{\prime+}(-y)(\beta+y+z) \\
\leq & V(-\beta)-g^{\prime+}(\beta)(\beta+y+z) \leq K_{t}+V(y+z)
\end{aligned}
$$

because $g^{\prime+}(-y) \geq g^{\prime+}(\beta), y+z \geq-\beta$ and we can use Lemma 5.1. So (5.4) is proved. 
(iv) $y-b<-\beta<y \leq y+z$. In this case we have $0<y+\beta<b$ and $r(y+z)=V(y+z), r(y)=V(y), r(y-b)=V(-\beta)+g(-(y-b))$, which means that it is sufficient to check

$$
K_{t}+V(y+z) \geq V(y)+z \frac{V(y)-V(-\beta)-g(b-y)+g(\beta)}{b} .
$$

For the proof of (5.5) consider two opposite subcases:

(a)

$$
\begin{aligned}
& \frac{V(y)-V(-\beta)}{y+\beta} \geq \frac{V(y)-V(-\beta)+g(\beta)-g(b-y)}{b}, \\
& \frac{V(y)-V(-\beta)}{y+\beta}<\frac{V(y)-V(-\beta)+g(\beta)-g(b-y)}{b} .
\end{aligned}
$$

(b)

Case (a). $V$ is $K_{t}$-convex. Thus

$$
\begin{aligned}
K_{t}+V(y+z) & \geq V(y)+z \frac{V(y)-V(-\beta)}{y+\beta} \\
& \geq V(y)+z \frac{V(y)-V(-\beta)+g(\beta)-g(b-y)}{b} .
\end{aligned}
$$

Case (b). The inequality (b) implies that

$$
\frac{b(V(y)-V(-\beta))-(y+\beta)(V(y)-V(-\beta))}{b(y+\beta)}<\frac{g(\beta)-g(b-y)}{b}
$$

and so

$$
V(y)<V(-\beta)+\frac{(y+\beta)(g(\beta)-g(b-y))}{b-y-\beta} ;
$$

this implies that

$$
\begin{aligned}
V(y)+z \frac{V(y)-V(-\beta)+g(\beta)-g(b-y)}{b} \\
\leq V(-\beta)+\frac{(y+\beta)(g(\beta)-g(b-y))}{b-y-\beta} \\
\quad+\frac{z}{b} \frac{(y+\beta)(g(\beta)-g(b-y))+(b-y-\beta))(g(\beta)-g(b-y))}{b-y-\beta} \\
=V(-\beta)+\frac{(y+\beta+z)(g(\beta)-g(b-y))}{b-y-\beta} \\
\leq V(-\beta)+\frac{(y+\beta+z)\left(-g^{\prime+}(\beta)\right)(b-y-\beta)}{b-y-\beta} \\
=V(-\beta)-g^{\prime+}(\beta)(y+\beta+z) .
\end{aligned}
$$

Since $y+z \geq-\beta$, by Lemma 5.1 we have $V(-\beta)-g^{\prime+}(\beta)(y+\beta+z) \leq$ $K_{t}+V(y+z)$, which proves (5.5). This finishes the proof of Proposition 5.1 and so of Theorem 4.1. 


\section{References}

[1] A. Bensoussan, M. Crouhy and J. M. Proth, Mathematical Theory of Production Planning, North-Holland, Amsterdam, 1983.

[2] D. Bertsekas, Dynamic Programming and Stochastic Control, Academic Press, 1976.

[3] S. Bylka, Algorithm for turnpike policies in the dynamic lot size model, Appl. Math. (Warsaw) 24 (1996), 57-75.

[4] A. Chikan (ed.), Inventory Models, Kluwer, 1990.

[5] R. Rempała, An aspect of $(s, S)$ inventory policy, in: Inventory Modelling, Vol. I, L. Bogataj (ed.), International Society for Inventory Research and University of Ljubljana, 1995, 15-21.

[6] H. Scraf, The optimality of $(S, s)$ policies in the dynamic inventory problem, in: Mathematical Methods in the Social Sciences, K. J. Arrow, S. Karlin and P. Suppes (eds.), Stanford University Press, 1960, Chapter 13.

[7] J. Zabczyk, Stochastic control of discrete-time systems, in: Control Theory and Topics in Functional Analysis, Vol. III, International Atomic Energy Agency, Vienna, $1976,187-223$.

Ryszarda Rempała

Institute of Mathematics

Polish Academy of Sciences

P.O. Box 137

00-950 Warszawa, Poland

E-mail: ryszrem@impan.gov.pl 